\title{
THE SMALL-SCALE PHOTOSPHERIC MAGNETIC FIELD AS AN INDICATOR OF THE DYNAMO
}

\author{
K. PETROVAY AND G. SZAKÁLY \\ Eötvös University, Department of Astronomy, Budapest, Ludovika tér 2, \\ H-1083 Hungary
}

\begin{abstract}
The presently widely accepted view that the solar dynamo operates near the base of the convective zone makes it difficult to relate the magnetic fields observed in the solar atmosphere to the fields in the dynamo layer. The large amount of observational data concerning photospheric magnetic fields could in principle be used to impose constraints on dynamo theory, but in order to infer these constraints the above mentioned "missing link" between the dynamo and surface fields should be found. This paper proposes such a link by modeling the passive vertical transport of thin magnetic flux tubes through the convective zone.
\end{abstract}

\section{INTRODUCTION}

Magnetic fields are observed to emerge into the photosphere in a preconcentrated form as loops formed from strong magnetic flux tubes (Stenflo 1989, Martin 1990). The crossing points of the loops with the photosphere appear as bipolar magnetic regions; according to the value of their $\Phi$ flux, the emerging fields are classified in three classes. (a) Active regions (ARs, $\Phi \sim 10^{13}-10^{14} \mathrm{~Wb}$ ) strictly obey Hale's polarity rules and their latitude-time distribution clearly delineates the equatorial branch of the butterfly diagram. After the decay of ARs, their magnetic fields diffuse out to form the unipolar network. (b) Ephemeral active regions (ERs, $\Phi \sim 10^{11}-10^{12} \mathrm{~Wb}$ ) obey Hale's polarity rules in a statistical sense and their latitude-time distribution diffusely delineates both the equatorial and polar branches of the butterfly diagram. After the decay of ERs, their magnetic fields form the mixed polarity network. (c) Intranetwork fields (IN fields, $\Phi \sim 10^{8}-10^{10} \mathrm{~Wb}$ ) - their orientation and distribution shows no obvious regularities. IN field elements decay to sizes under the resolution boundary before they could reach the supergranule boundaries, so they only give a minor contribution to the mixed network.

The rate of flux emergence in the form of ARs:ERs:IN fields is 1:100: $10^{4}$ (Zirin 1987). This enormous difference is however largely compensated by the very different lifetimes of these flux concentrations, so according to a study by Martin (1990) the contribution to the total unsigned flux density $\langle\mathbf{B} \mid\rangle$ crossing the solar surface is similar for ERs (+ mixed network) and IN fields (around $0.5 \mathrm{mT}$ ). The contribution by ARs depends strongly on solar cycle phase, but in general it is also of similar magnitude. 
In order to forge a link between magnetic fields in the photosphere and those in the dynamo region, a thorough understanding of the mechanisms of magnetic field transport in the convective zone is needed. Efforts in this direction have always concentrated on the problem of the origin of active regions, neglecting the problem of the smaller scale fields. Studying the transport of small-scale fields through the convective zone is however just as important as the investigation of the origin of active regions.

From the theoretical point of view the sole basis of the difference between the transport of thick (AR size) and thin (IN size) magnetic flux tubes in a stratified turbulent medium is their different surface/volume ratio. The magnetic flux density in flux tubes in a strongly turbulent medium is known to be order of the $B_{e}$ equipartitional flux density for which $B_{e}^{2} / 2 \mu=\rho v^{2} / 2$ ( $\rho$ is the density, $v$ the turbulent velocity, $\mu$ the magnetic permeability). Among the forces acting on the tube, the buoyancy $F_{b} \sim B^{2} / 2 \mu_{0} H_{P} \sim \rho v^{2} / 2 H_{P}\left(H_{P}\right.$ : pressure scale height) and the curvature force $F_{m} \sim B^{2} / \mu_{0} l \sim \rho v^{2} / l$ (l: correlation length) are of similar order of magnitude if $l \sim H_{P}$, while the drag, acting on the surface of the tube, is $F_{d} \sim \rho v^{2} / d$ (d: tube diameter). Clearly, for thin tubes (with $d \ll H_{P}$ ) the drag will dominate, while for thick tubes the volume forces (buoyancy and curvature) will dominate. Consequently, the thin tubes will be passively transported by the turbulence. This paper is a brief summary of our at tempt to model the transport and the resulting equilibrium distribution of these "passive" fields; more details will be presented in an upcoming paper (Petrovay and Szakály 1992).

The observational constraints the model has to comply with are the following. As mentioned above, Martin (1990) has found that the observed unsigned flux density of IN fields in the photosphere is about $0.5 \mathrm{mT}$. According to Stenflo (1989) the "hidden" part of the turbulent flux (below the resolution threshold) may be between 1 and $10 \mathrm{mT}$, so the models must have $\langle|\mathrm{B}|\rangle=0.5-10 \mathrm{mT}$ at the photosphere. The signed flux density $\langle\mathbf{B}\rangle$ (i.e. the large-scale field) has an r.m.s. value of $0.15 \mathrm{mT}$. The large-scale field varies by a factor of 3 between solar minimum and maximum, so the joint contribution of IN fields and ERs to the signed flux density may be about $0.05 \mathrm{mT}$. How much of this is due to the IN fields is not known, but constraining the r.m.s. $\langle\mathbf{B}\rangle$ to the regime $0.01-0.05 \mathrm{mT}$ is probably realistic. This large-scale field is thought to be mainly radial in the photosphere because of the strong buoyancy there, this effect is however probably limited to a shallow surface layer (of depth $<100 \mathrm{~km}$ ) only, below which the r.m.s. $\left\langle B_{x}\right\rangle$ is comparable to the the r.m.s. $\left\langle B_{z}\right\rangle(x, z$ horizontal and vertical coordinates, respectively, and the r.m.s. values taken over the whole solar surface).

These two observational constraints lead us to study the transport of $\langle\mathbf{B}\rangle$ and $\langle|\mathbf{B}|\rangle$ separately.

\section{EQUATIONS AND RESULTS}

In a one-dimensional, plane parallel model with $z$ the depth our transport equation for $\langle\mathbf{B}\rangle$ is

$$
0 \equiv \frac{\partial\left\langle B_{x}\right\rangle}{\partial t}=\frac{d}{d z}\left(\beta \frac{d\left\langle B_{x}\right\rangle}{d z}\right)+\frac{d}{d z}\left(\gamma\left\langle B_{x}\right\rangle\right)
$$


in the case of equilibrium. The $\beta$ turbulent magnetic diffusivity is $\frac{1}{3} v l$ for isotropic turbulence. The convective zone model we will use here (Unno, Kondo and Xiong 1985) assumes anisotropic turbulence with $\overline{v_{z}^{2}} / \overline{v^{2}}=0.5$; in this case $\beta=v l / 2$. In the same way, $-\gamma=-\tau \nabla \overline{v^{2}} / 2$ for the turbulent pumping velocity $(\tau=l / v)$.

The transport equation of $\langle|\mathrm{B}|\rangle$ will be formally similar to (1) with an additional term describing the small scale dynamo action:

$$
0 \equiv \frac{\partial\langle|\mathbf{B}|\rangle \mid}{\partial t}=\frac{d}{d z}\left(\beta \frac{d\langle|\mathbf{B}|\rangle}{d z}\right)+\frac{d}{d z}(\gamma\langle|\mathbf{B}|\rangle)+\left(1-\langle|\mathbf{B}|\rangle / f_{l} B_{e}\right)\langle|\mathbf{B}|\rangle v / l .
$$

with $f_{l}=0.1$.

A detailed discussion of these equations is given in Petrovay and Szakály (1992). Further source terms may arise both in (1) and (2) if the interaction of "active" and "passive" fields is taken into account, i.e. the decay of active, buoyant, thick tubes at different levels in the convective zone may resupply the passively transported field.
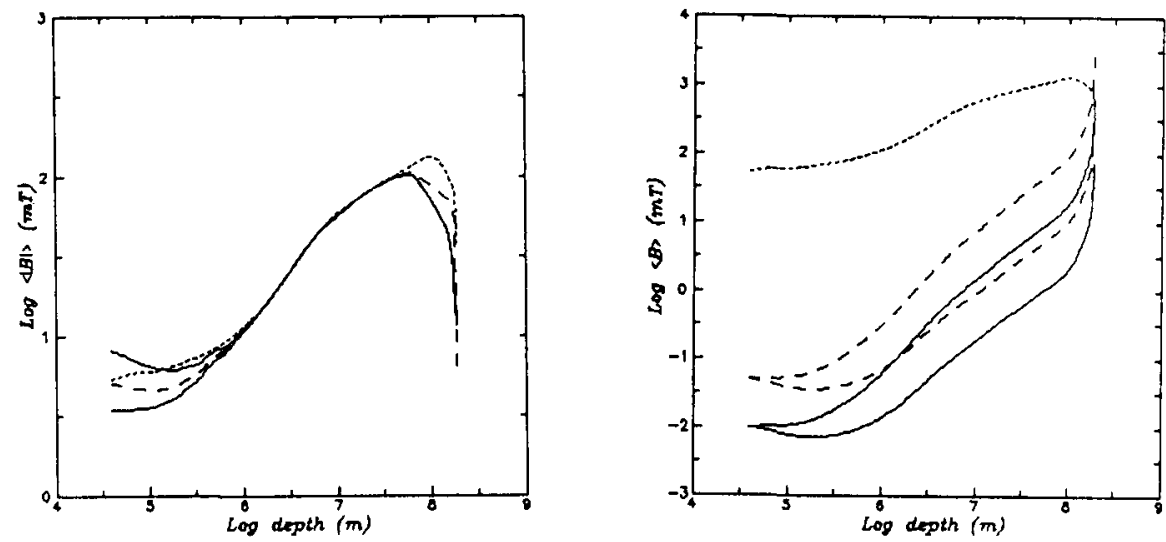

FIGURE I Unsigned flux density $\langle|\mathbf{B}|\rangle$ as a function of depth. Solid curves: $L=0$ at bottom, $L=0$ (upper curve) $/ L=\max$. (lower curve) at top. Long dashes: $L=$ medium at top, $L=0$ (lower curve) $/-L=$ max (upper curve) at bottom. Short dashes: saturation flux density of small-scale dynamo.

FIGURE II R. m. s. mean flux density $\left\langle B_{x}\right\rangle$ as a function of depth. Solid curves: $B_{x}=0.01 \mathrm{mT}, L=0$ (lower curve) $/ L=\max$. (upper curve) at top. Long dashes: $B_{x}=0.05 \mathrm{mT}, L=0$ (lower curve) $/ L=\max$. (upper curve) at top. Short dashes: equipartition field.

The results of a numerical solution of Equation (2) are shown on Figure I. The resulting value of $\langle|\mathbf{B}|\rangle$ in the photosphere depends only on the upper boundary conditions applied and is not sensitive at all to the lower boundary conditions (or, indeed, to the whole behavior of the solution in the lower half of the convective zone). If the upper boundary condition is specified as $\frac{d^{2}}{d z^{2}}\langle|\mathrm{~B}|\rangle=0$ 
or as $L \equiv \beta \frac{d}{d z}\langle|B|\rangle+\gamma\langle|\mathbf{B}|\rangle=0$ then $\langle|\mathbf{B}|\rangle_{p h o t} \sim 3 \mathrm{mT}$. In the other extreme when $L=v B_{\text {phat }},\langle|\mathbf{B}|\rangle_{\text {phot }} \sim 8 \mathrm{mT}$. These results agree well with the observational constraints given at the end of Section 1; indeed, they constrain the value of the hidden flux density to between 2.5 and 7.5 .

On the other hand, if the small-scale dynamo term is omitted in (4), the solution is able to reproduce the surface value of the unsigned flux density $(>0.5 \mathrm{mT})$ only if in the lower convective zone $\langle|\mathrm{B}|\rangle$ exceeds $B_{e}$ by several orders of magnitude. This can be excluded as then convection would be inhibited. A strong source term in equation (4) is therefore needed to reproduce the observed photospheric unsigned intranetwork flux density. The observed flux emergence rate in ARs and ERs is also much lower than the necessary source, so (unless we make the rather implausible assumption that the dispersal of "active" fields yields a source of the passive fields that is by several orders of magnitude stronger than that observed in the photosphere) we are forced to assume the operation of a small-scale dynamo in the solar convective zone.

Now let us turn to the problem of the transport of the mean field. Equation (1) can be directly integrated to yield

$$
\beta \frac{d\left\langle B_{x}\right\rangle}{d z}+\left(\gamma\left\langle B_{x}\right\rangle\right)=L
$$

Solving (3) with the same choices for $L$ as before and with the limits for the near-photospheric r.m.s. $\left\langle B_{x}\right\rangle$ of passive origin quoted in Section 1 as boundary conditions, the resulting $\left\langle B_{x}\right\rangle(z)$ functions are shown on Figure II. It is apparent that, owing to the strong turbulent pumping downward, the equilibrium distribution is very steep: the surface mean field is just the "tip of the iceberg". In fact, nearly all the large-scale field is concentrated near the bottom of the convective zone, confirming the old guess (Schüßler 1984) that turbulent pumping is the main agent confining the dynamo to the base of the convective zone.

\section{ACKNOWLEDGMENTS}

This work was funded by the OTKA (grants no. 2135 and F 4342) as well as by the Hungarian Science Foundation (grant no. 23/91/III) and Hungarian High Education Foundation (grant no. 69/91) of the Hungarian Credit Bank.

\section{REFERENCES}

Martin, S. 1990, Proc. IAU Symp. 138, 129.

Petrovay, K. and Szakály, G. 1992, Astr. Ap., submitted

Schüßler, M. 1984, in: The Hydromagnetics of the Sun, Proc. Fourth Eur. Meeting on Solar Phys. , ESA, Paris

Stenflo, J. O. 1989, Astr. Ap. Rev., 1, 3.

Unno, W., Kondo, M. and Xiong, D.-R. 1985, P. A. S. Japan, 37, 235.

Zirin, H. 1987, Solar Phys., 110, 101. 